\title{
The controllability of damped fractional differential system with impulses and state delay
}

\author{
Musarrat Nawaz $^{1 *} \mathbb{D}$, Jiang Wei ${ }^{1 *}$, Jiale Sheng ${ }^{1}$ and Azmat Ullaha Khan ${ }^{1}$
}

\section{"Correspondence:}

nawazmusarrat@yahoo.com; jiangwei@ahu.edu.cn

'School of Mathematical Sciences, Anhui University, Hefei, China

\section{Springer}

\begin{abstract}
We discuss the controllability for a damped fractional differential system with impulses and state delay, which involves Caputo fractional derivatives. Deriving the condition based on the Gramian matrix defined by the Mittag-Leffler matrix function and Laplace transformation, we establish necessary and sufficient conditions of controllability criteria. Finally, we construct two numerical examples to support the result.
\end{abstract}

MSC: $34 \mathrm{~K} 05 ; 26 \mathrm{~A} 33$

Keywords: Fractional differential equations; Controllability; State delay; Impulses

\section{Introduction}

The fractional differential equations have proven to be a useful tool for modeling distinct phenomena in various fields of physics, engineering, and economics. Various practical systems can be described more accurately through fractional derivative formulation, as explained in detail based on the theory of fractional calculus [1-6]. Many mathematicians, engineers, and physicists contributed in fractional differential equations theory and its applications. Fractional derivatives have several kinds, such as Caputo, Riemann-Liouville, Grunwald-Letnikov, and Hadamard. Nowadays research on fractional delay differential equations is on the rise, whereas the theory of delay differential equations is well developed [7-15].

The controllability performs an important role in the formulation of current mathematical control theory and engineering, which has a close connection with structural decomposition, quadratic optimal, and so on [16-21]. The controllability is a subjective property in the theory of dynamical systems [22-24]. The controllability of linear systems, nonlinear systems, and stochastic systems with delay has been investigated in [25-31]. Moreover, in recent years, controllability problems for linear and nonlinear fractional differential system are discussed by Matar and Nawaz [32-35], and partial controllability of various semilinear systems is discussed in [36-45]. More recent research work on controllability of various impulsive systems is reported in [46-57].

(c) The Author(s) 2020. This article is licensed under a Creative Commons Attribution 4.0 International License, which permits use sharing, adaptation, distribution and reproduction in any medium or format, as long as you give appropriate credit to the original author(s) and the source, provide a link to the Creative Commons licence, and indicate if changes were made. The images or other third party material in this article are included in the article's Creative Commons licence, unless indicated otherwise in a credit line to the material. If material is not included in the article's Creative Commons licence and your intended use is not permitted by statutory regulation or exceeds the permitted use, you will need to obtain permission directly from the copyright holder. To view a copy of this licence, visit http://creativecommons.org/licenses/by/4.0/. 
Many dynamical systems have been studied by applying discrete- or continuous-time domains. Moreover, many real systems in biology, physics, chemistry, information science, and engineering may face sudden changes at certain instants during continuous dynamical processes. This type of behavior can be formed by impulsive systems. In the past few years the research on impulsive control systems has aroused magnificent interest; the existence of impulses can be observed in biological phenomena containing bursting rhythm models in biology and medicine, thresholds, and frequency-modulated systems. The basic knowledge of a impulsive differential equations can be found in the monographs of Benchohra et al. [58] and Bainov et al. [59]. Feickan et al. [60] discussed the concept and existence of solutions for impulsive fractional differential equations. The controllability and observability for linear fractional impulsive systems with time-invariance are discussed in [61]. Furthermore, the controllability criteria for linear and nonlinear fractional differential systems with state delay and impulses is studied in [62,63]. The controllability of second-order semilinear impulsive stochastic neutral functional evolution equations is explained in [64]. In addition, the controllability of impulsive neutral functional differential inclusions in Banach spaces is studied by Wan et al. [65]. Moreover, a recent study on neural networks via impulsive control is discussed in [66]. The derivation of the condition in the present study is based on analytical evaluation of the Gramian matrix defined by the Mittage-Leffler matrix function. However, from a numerical point of view, the MittagLeffler matrix function is explained by Garrappa [67]. The study of the damped fractional differential system for its controllability results by using the Mittag-Leffler matrix function and Gramian matrix is presented in [68]. Moreover, the Kalman rank criterion is obtained for the controllability of fractional impulse controlled systems in [69], where controllability is dependent on the impulses.

The previous studies mainly focused on different types of fractional impulsive systems. Still no work is reported on the controllability of the damped fractional differential system with impulses and state delay. In this paper, motivated mainly by $[62,68,70]$, in this study, we are concerned with the controllability criteria for the damped fractional differential system with impulses and algebraic-based state delay. This paper is organized as follows. Section 2 includes a few fundamental definitions, preliminary results, and lemmas to prove the controllability of a damped fractional differential system with impulses and state delay. In Sect. 3, we obtain a sufficient condition for the controllability of a damped fractional differential system with impulses and state delay by step technique. Two examples in Sect. 4 demonstrate the applicability of the outcomes.

In this study, we consider the controllability of damped fractional differential systems with impulses and state delay

$$
\left\{\begin{array}{l}
{ }^{c} D^{\gamma} x(t)-{ }^{c} D^{\delta} A x(t)=B x(t-\tau)+C u(t), \quad t \in[0, T] \backslash\left\{t_{1}, t_{2}, \ldots, t_{k}\right\} \\
\Delta x\left(t_{i}\right)=x\left(t_{i}^{+}\right)-x\left(t_{i}^{-}\right)=I_{i}\left(x\left(t_{i}\right)\right), \quad i=1,2,3, \ldots, k, \\
x(t)=\varphi(t), \quad t \in[-\tau, 0], \\
x^{\prime}(t)=\varphi^{\prime}(t),
\end{array}\right.
$$

where ${ }^{c} D^{\gamma} x(t)$ and ${ }^{c} D^{\delta} x(t)$ denote the $\gamma$ th- and $\delta$ th-order Caputo fractional derivatives of $x(t), 0<\delta<1<\gamma<2, x(t) \in \mathcal{R}^{n}$ is the state vector, $u(t) \in \mathcal{R}^{m}$ is the control vector, $A$, $B \in \mathcal{R}^{n \times n}$ and $C \in \mathcal{R}^{n \times m}$ are any matrices, $\tau$ is a positive constant, and $\varphi(t) \in C\left([-\tau, 0], \mathcal{R}^{n}\right)$ 
is the function that represents the initial state, where $C\left([-\tau, 0], \mathcal{R}^{n}\right)$ is the space of all continuous functions from the interval $[-\tau, 0]$ into $\mathcal{R}^{n}, I_{i}: \mathcal{R}^{n} \rightarrow \mathcal{R}^{n}$ is continuous for $i=1,2,3, \ldots, k$, where $k$ is an integer,

$$
x\left(t_{i}^{+}\right)=\lim _{\varepsilon \rightarrow 0^{+}} x\left(t_{i}+\varepsilon\right), \quad x\left(t_{i}^{-}\right)=\lim _{\varepsilon \rightarrow 0^{-}} x\left(t_{i}+\varepsilon\right)
$$

represent the right and left limits of $x(t)$ at the discontinuity points $t=t_{i}$,

$$
t_{i-1}<i \tau<t_{i}, \quad i=1,2, \ldots, k,
$$

where $0=t_{0}$ and $t_{k+1}=T$, and $x\left(t_{i}\right)=x\left(t_{i}^{-}\right)$, which means that the solution of system (1) is left continuous at $t_{i}$.

\section{Preliminaries and essential lemmas}

We denote by $C_{p}\left([0, T], \mathcal{R}^{n}\right)$ the space of all piecewise left-continuous functions from the interval $[0, T]$ into $\mathcal{R}^{n}$.

To obtain the main results, we introduce some fundamental definitions and lemmas. The following basic definitions are well known; for more detail, see [1-5].

Definition 2.1 The Riemann-Liouville fractional integral of order $\gamma>0$ for a function $f: R^{+} \rightarrow R$ is defined as

$$
D^{-\gamma} f(t)=\frac{1}{\Gamma(\gamma)} \int_{0}^{t}(t-\theta)^{\gamma-1} f(\theta) d \theta
$$

where $\Gamma$ is the Euler gamma function.

Definition 2.2 ([14]) The Caputo fractional derivative of order $\gamma(0 \leq m<\gamma \leq m+1)$ for a function $f: R^{+} \rightarrow R^{n}$ is defined as

$$
{ }^{c} D^{\gamma} f(t)=\frac{1}{\Gamma(m-\gamma+1)} \int_{0}^{t} \frac{f^{(m+1)}(\theta)}{(t-\theta)^{\gamma-m}} d \theta .
$$

The Laplace transform of the Caputo fractional derivative is

$$
\begin{aligned}
& L\{f(t) ;(s)\}=F(s), \\
& L\left\{{ }^{c} D^{\gamma} f(t)\right\}(s)=s^{\gamma} F(s)-\sum_{k=0}^{m-1} f^{(k)}\left(0^{+}\right) s^{\gamma-1-k} .
\end{aligned}
$$

In particular, if $0<\gamma \leq 1$, then

$$
L\left\{{ }^{c} D^{\gamma} f(t)\right\}(s)=s^{\gamma} F(s)-f\left(0^{+}\right) s^{\gamma-1},
$$

and if $1<\gamma \leq 2$, then

$$
L\left\{{ }^{c} D^{\gamma} f(t)\right\}(s)=s^{\gamma} F(s)-f\left(0^{+}\right) s^{\gamma-1}-f\left(0^{+}\right) s^{\gamma-2},
$$

where $s$ is the complex spectral variable of the Laplace transform. 
Definition 2.3 Consider the Mittag-Leffler function

$$
E_{\gamma, \delta}(z)=\sum_{k=0}^{\infty} \frac{z^{k}}{\Gamma(\gamma k+\delta)}, \quad z \in C, \gamma>0, \delta>0
$$

where $C$ is the complex plane. For $\delta=1$, the Mittag-Leffler function becomes

$$
E_{\gamma}\left(\lambda z^{\gamma}\right)=E_{\gamma, 1}\left(\lambda z^{\gamma}\right)=\sum_{k=0}^{\infty} \frac{\lambda^{k} z^{k \gamma}}{\Gamma(\gamma k+1)}, \quad \lambda, z \in C
$$

The Laplace transform of the Mittag-Leffler function is

$$
L\left\{t^{\delta-1} E_{\gamma, \delta}\left( \pm a t^{\gamma}\right)\right\}(s)=\frac{s^{\gamma-\delta}}{\left(s^{\gamma} \mp a\right)} .
$$

Particularly, for $\delta=1$,

$$
L\left\{E_{\gamma}\left( \pm a t^{\gamma}\right)\right\}(s)=\frac{s^{\gamma-1}}{\left(s^{\gamma} \mp a\right)} .
$$

Lemma 2.4 ([5]) For any $\gamma, \delta>0$ and $A \in C^{n \times n}$, we have

$$
L\left\{t^{\delta-1} E_{\gamma, \delta}\left( \pm A t^{\gamma}\right)\right\}(s)=s^{\gamma-\delta}\left(s^{\gamma} I \mp A\right)^{-1}, \quad \Re(s)>\|A\|^{\frac{1}{a}},
$$

where $\Re(s)$ is the real part of a complex number s, and I is the identity matrix.

First, we consider the representation of solutions of damped fractional delay differential systems without impulses to obtain the state response of system (1) as follows:

$$
\left\{\begin{array}{l}
{ }^{c} D^{\gamma} x(t)-{ }^{c} D^{\delta} A x(t)=B x(t-\tau)+f(t), \quad t \in[0, T], \\
x(t)=\varphi(t), \quad t \in[-\tau, 0] .
\end{array}\right.
$$

Lemma 2.5 Let $0<\delta<1<\gamma<2$. If $f:[0, T] \rightarrow \mathcal{R}^{n}$ is continuous and exponentially bounded, then the solution of (4) is equivalent to the solution of the system

$$
\left\{\begin{aligned}
x(t)= & \varphi(0)+t E_{\gamma-\delta, 2}\left(A t^{\gamma-\delta}\right) \varphi^{\prime}(0)+\int_{0}^{t}(t-s)^{\gamma-1} E_{\gamma-\delta, \gamma}\left[A(t-s)^{\gamma-\delta}\right] \\
& \times[B x(s-\tau)+f(s)] d s, \quad t \in[0, T], \\
\varphi(0)+ & t E_{\gamma-\delta, 2}\left(A t^{\gamma-\delta}\right) \varphi^{\prime}(0) \\
& +\sum_{j=2}^{n} \int_{(j-2) \tau}^{(j-1) \tau}(t-s)^{\gamma-1} E_{\gamma-\delta, \gamma}\left[A(t-s)^{\gamma-\delta}\right]\left[B \varphi_{j-2}(s-\tau)\right] d s \\
& +\int_{(j-1) \tau}^{t}(t-s)^{\gamma-1} E_{\gamma-\delta, \gamma}\left[A(t-s)^{\gamma-\delta}\right]\left[B \varphi_{j-1}(s-\tau)\right] d s \\
& +\int_{0}^{t}(t-s)^{\gamma-1} E_{\gamma-\delta, \gamma}\left[A(t-s)^{\gamma-\delta}\right] f(s) d s, \quad t \in[(i-1) \tau, i \tau], i=1,2, \ldots, n, \\
x(t) & =\varphi(t), \quad t \in[-\tau, 0] .
\end{aligned}\right.
$$

Proof By implementing the step technique in [71] there is a unique solution to system (4). For $t \in[0, T]$, by taking the Laplace transform with respect to $t$ on both sides of system (4) we get

$$
S^{\gamma}[L x(t)]-s^{\gamma-1} x(0)-s^{\gamma-2} x^{\prime}(0)-A s^{\delta}[L x(t)]+A s^{\delta-1} x(0)=L[B x(t-\tau)+f(t)],
$$




$$
\begin{aligned}
{[L x(t)]=} & \left(s^{\gamma} I-A s^{\delta}\right)^{-1} s^{\gamma-1} \varphi(0) \\
& -A\left(s^{\gamma} I-A s^{\delta}\right)^{-1} s^{\delta-1} \varphi(0)+\left(s^{\gamma} I-A s^{\delta}\right)^{-1} s^{\gamma-2}\left(\varphi^{\prime}(0)\right) \\
& +\left(s^{\gamma} I-A s^{\delta}\right)^{-1} L[B x(t-\tau)+f(t)], \\
= & \left(s^{\gamma} I-A s^{\delta}\right)^{-1} s^{\gamma} L[\varphi(0)] \\
& -A\left(s^{\gamma} I-A s^{\delta}\right)^{-1} s^{\delta} L[\varphi(0)]+\left(s^{\gamma} I-A s^{\delta}\right)^{-1} s^{\gamma-2}\left(\varphi^{\prime}(0)\right) \\
& +\left(s^{\gamma} I-A s^{\delta}\right)^{-1} L[B x(t-\tau)+f(t)], \\
= & L[\varphi(0)]+A\left(s^{\gamma-\delta} I-A\right)^{-1} L[\varphi(0)] \\
& -A\left(s^{\gamma-\delta} I-A\right)^{-1} L[\varphi(0)]+\left(s^{\gamma-\delta} I-A\right)^{-1} s^{\gamma-\delta-2}\left(\varphi^{\prime}(0)\right) \\
& +\left(s^{\gamma-\delta} I-A\right)^{-1} s^{-\delta} L[B x(t-\tau)+f(t)], \\
{[L x(t)]=} & L[\varphi(0)]+L\left[t E_{\gamma-\delta, 2}\left(A t^{\gamma-\delta}\right)\right] \varphi^{\prime}(0) \\
& +L\left[t^{\gamma-1} E_{\gamma-\delta, \gamma}\left(A t^{\gamma-\delta}\right)\right] \times[B x(t-\tau)+f(t)] .
\end{aligned}
$$

Now by applying the convolution theorem on (7) we get

$$
\begin{aligned}
L[x(t)]= & L[\varphi(0)]+L\left[t E_{\gamma-\delta, 2}\left(A t^{\gamma-\delta}\right) \varphi^{\prime}(0)\right] \\
& +L \int_{0}^{t}(t-s)^{\gamma-1} E_{\gamma-\delta, \gamma}\left[A(t-s)^{\gamma-\delta}\right][B x(s-\tau)+f(s)] d s .
\end{aligned}
$$

Applying the inverse Laplace transform to equation (8), we get

$$
\begin{aligned}
x(t)= & \varphi(0)+t E_{\gamma-\delta, 2}\left(A t^{\gamma-\delta}\right) \varphi^{\prime}(0) \\
& +\int_{0}^{t}(t-s)^{\gamma-1} E_{\gamma-\delta, \gamma}\left[A(t-s)^{\gamma-\delta}\right][B x(s-\tau)+f(s)] d s, \quad t \in[0, T] .
\end{aligned}
$$

Denote $x(t)=\varphi(t)=\varphi_{0}(t), t \in[-\tau, 0]$. Then for $t \in[0, \tau]$,

$$
\begin{aligned}
x(t)= & \varphi(0)+t E_{\gamma-\delta, 2}\left(A t^{\gamma-\delta}\right) \varphi^{\prime}(0) \\
& +\int_{0}^{t}(t-s)^{\gamma-1} E_{\gamma-\delta, \gamma}\left[A(t-s)^{\gamma-\delta}\right]\left[B \varphi_{0}(s-\tau)+f(s)\right] d s, \quad t \in[0, \tau] .
\end{aligned}
$$

Denote $\varphi_{1}(t)=x(t), t \in[0, \tau]$. Then for $t \in[\tau, 2 \tau]$,

$$
\begin{aligned}
x(t)= & \varphi(0)+t E_{\gamma-\delta, 2}\left(A t^{\gamma-\delta}\right) \varphi^{\prime}(0)+\int_{0}^{\tau}(t-s)^{\gamma-1} E_{\gamma-\delta, \gamma}\left[A(t-s)^{\gamma-\delta}\right] \\
& \times\left[B \varphi_{0}(s-\tau)\right] d s+\int_{\tau}^{t}(t-s)^{\gamma-1} E_{\gamma-\delta, \gamma}\left[A(t-s)^{\gamma-\delta}\right]\left[B \varphi_{1}(s-\tau)\right] d s \\
& +\int_{0}^{t}(t-s)^{\gamma-1} E_{\gamma-\delta, \gamma}\left[A(t-s)^{\gamma-\delta}\right] f(s) d s .
\end{aligned}
$$


Denote $\varphi_{2}(t)=x(t), t \in[\tau, 2 \tau]$. Then for $t \in[2 \tau, 3 \tau]$,

$$
\begin{aligned}
x(t)= & \varphi(0)+t E_{\gamma-\delta, 2}\left(A t^{\gamma-\delta}\right) \varphi^{\prime}(0)+\int_{0}^{\tau}(t-s)^{\gamma-1} E_{\gamma-\delta, \gamma}\left[A(t-s)^{\gamma-\delta}\right] \\
& \times\left[B \varphi_{0}(s-\tau)\right] d s+\int_{\tau}^{2 \tau}(t-s)^{\gamma-1} E_{\gamma-\delta, \gamma}\left[A(t-s)^{\gamma-\delta}\right]\left[B \varphi_{1}(s-\tau)\right] d s \\
& +\int_{2 \tau}^{t}(t-s)^{\gamma-1} E_{\gamma-\delta, \gamma}\left[A(t-s)^{\gamma-\delta}\right]\left[B \varphi_{2}(s-\tau)\right] d s \\
& +\int_{0}^{t}(t-s)^{\gamma-1} E_{\gamma-\delta, \gamma}\left[A(t-s)^{\gamma-\delta}\right] f(s) d s .
\end{aligned}
$$

Denote $x(t)=\varphi_{n}(t), t \in[(n-2) \tau,(n-1) \tau]$. Then for $t \in[(n-1) \tau, n \tau]=[(n-1) \tau, T]$,

$$
\begin{aligned}
& x(t)= \varphi(0)+t E_{\gamma-\delta, 2}\left(A t^{\gamma-\delta}\right) \varphi^{\prime}(0)+\int_{0}^{\tau}(t-s)^{\gamma-1} E_{\gamma-\delta, \gamma}\left[A(t-s)^{\gamma-\delta}\right] \\
& \times\left[B \varphi_{0}(s-\tau)\right] d s+\int_{\tau}^{2 \tau}(t-s)^{\gamma-1} E_{\gamma-\delta, \gamma}\left[A(t-s)^{\gamma-\delta}\right]\left[B \varphi_{1}(s-\tau)\right] d s+\cdots \\
&+\int_{(n-2) \tau}^{(n-1) \tau}(t-s)^{\gamma-1} E_{\gamma-\delta, \gamma}\left[A(t-s)^{\gamma-\delta}\right]\left[B \varphi_{n-2}(s-\tau)\right] d s \\
&+\int_{(n-1) \tau}^{t}(t-s)^{\gamma-1} E_{\gamma-\delta, \gamma}\left[A(t-s)^{\gamma-\delta}\right]\left[B \varphi_{n-1}(s-\tau)\right] d s \\
&+\int_{0}^{t}(t-s)^{\gamma-1} E_{\gamma-\delta, \gamma}\left[A(t-s)^{\gamma-\delta}\right] f(s) d s, \\
& x(t)=\left\{\begin{array}{c}
\varphi(0)+t E_{\gamma-\delta, 2}\left(A t^{\gamma-\delta}\right) \varphi^{\prime}(0) \\
\quad+\int_{0}^{t}(t-s)^{\gamma-1} E_{\gamma-\delta, \gamma}\left[A(t-s)^{\gamma-\delta}\right]\left[B \varphi_{0}(s-\tau)+f(s)\right] d s, \quad t \in[0, \tau], \\
\quad+\sum_{j=2}^{n} \int_{(j-2) \tau}^{(j-1) \tau}(t-s)^{\gamma-1} E_{\gamma-\delta, \gamma}\left[A(t-s)^{\gamma-\delta}\right]\left[B \varphi_{j-2}(s-\tau)\right] d s \\
\quad+\int_{(j-1) \tau}^{t}(t-s)^{\gamma-1} E_{\gamma-\delta, \gamma}\left[A(t-s)^{\gamma-\delta}\right]\left[B \varphi_{j-1}(s-\tau)\right] d s \\
+\int_{0}^{t}(t-s)^{\gamma-1} E_{\gamma-\delta, \gamma}\left[A(t-s)^{\gamma-\delta}\right] f(s) d s, \quad t \in[(i-1) \tau, i \tau], i=1,2, \ldots, n .
\end{array}\right.
\end{aligned}
$$

Lemma 2.6 Let $0<\delta<1<\gamma<2$ and $u \in C_{p}\left([0, T], \mathcal{R}^{m}\right)$. Then system (1) state response representation can be defined as follows. For $t \in[-\tau, 0]$,

$x(t)=\varphi(t) ;$

for $t \in\left[0, t_{1}\right]$,

$$
\begin{aligned}
x(t)= & \varphi(0)+t E_{\gamma-\delta, 2}\left(A t^{\gamma-\delta}\right) \varphi^{\prime}(0) \\
& +\int_{0}^{t}(t-s)^{\gamma-1} E_{\gamma-\delta, \gamma}\left[A(t-s)^{\gamma-\delta}\right]\left[B \varphi_{0}(s-\tau)+C u(s)\right] d s ;
\end{aligned}
$$


for $t \in\left(t_{1}, \tau\right]$,

$$
\begin{aligned}
x(t)= & \varphi(0)+I_{1}\left(x\left(t_{1}^{-}\right)\right)+t E_{\gamma-\delta, 2}\left(A t^{\gamma-\delta}\right) \varphi^{\prime}(0) \\
& +\int_{0}^{t}(t-s)^{\gamma-1} E_{\gamma-\delta, \gamma}\left[A(t-s)^{\gamma-\delta}\right]\left[B \varphi_{0}(s-\tau)+C u(s)\right] d s ;
\end{aligned}
$$

for $t \in\left(\tau, t_{2}\right]$,

$$
\begin{aligned}
x(t)= & \varphi(0)+I_{1}\left(x\left(t_{1}^{-}\right)\right)+t E_{\gamma-\delta, 2}\left(A t^{\gamma-\delta}\right) \varphi^{\prime}(0) \\
& +\int_{0}^{\tau}(t-s)^{\gamma-1} E_{\gamma-\delta, \gamma}\left[A(t-s)^{\gamma-\delta}\right] B \varphi_{0}(s-\tau) d s \\
& +\int_{\tau}^{t}(t-s)^{\gamma-1} E_{\gamma-\delta, \gamma}\left[A(t-s)^{\gamma-\delta}\right] B \varphi_{1}(s-\tau) d s \\
& +\int_{0}^{t}(t-s)^{\gamma-1} E_{\gamma-\delta, \gamma}\left[A(t-s)^{\gamma-\delta}\right] C u(s) d s ;
\end{aligned}
$$

for $t \in\left(t_{2}, 2 \tau\right]$,

$$
\begin{aligned}
x(t)= & \varphi(0)+I_{1}\left(x\left(t_{1}^{-}\right)\right)+I_{2}\left(x\left(t_{2}^{-}\right)\right)+t E_{\gamma-\delta, 2}\left(A t^{\gamma-\delta}\right) \varphi^{\prime}(0) \\
& +\int_{0}^{\tau}(t-s)^{\gamma-1} E_{\gamma-\delta, \gamma}\left[A(t-s)^{\gamma-\delta}\right] B \varphi_{0}(s-\tau) d s \\
& +\int_{\tau}^{t}(t-s)^{\gamma-1} E_{\gamma-\delta, \gamma}\left[A(t-s)^{\gamma-\delta}\right] B \varphi_{1}(s-\tau) d s \\
& +\int_{0}^{t}(t-s)^{\gamma-1} E_{\gamma-\delta, \gamma}\left[A(t-s)^{\gamma-\delta}\right] C u(s) d s,
\end{aligned}
$$

for $t \in\left((i-1) \tau, t_{i}\right], i \geq 2$,

$$
\begin{aligned}
x(t)= & \varphi(0)+\sum_{j=1}^{i-1} I_{j}\left(x\left(t_{j}^{-}\right)\right)+t E_{\gamma-\delta, 2}\left(A t^{\gamma-\delta}\right) \varphi^{\prime}(0) \\
& +\sum_{j=1}^{i-1} \int_{(j-1) \tau}^{(j) \tau}(t-s)^{\gamma-1} E_{\gamma-\delta, \gamma}\left[A(t-s)^{\gamma-\delta}\right]\left[B \varphi_{j-1}(s-\tau)\right] d s \\
& +\int_{(i-1) \tau}^{t}(t-s)^{\gamma-1} E_{\gamma-\delta, \gamma}\left[A(t-s)^{\gamma-\delta}\right]\left[B \varphi_{i-1}(s-\tau)\right] d s \\
& +\int_{0}^{t}(t-s)^{\gamma-1} E_{\gamma-\delta, \gamma}\left[A(t-s)^{\gamma-\delta}\right] C u(s) d s ;
\end{aligned}
$$

for $t \in\left(t_{i}, i \tau\right], i \geq 2$,

$$
\begin{aligned}
x(t)= & \varphi(0)+\sum_{j=1}^{i} I_{j}\left(x\left(t_{j}^{-}\right)\right)+t E_{\gamma-\delta, 2}\left(A t^{\gamma-\delta}\right) \varphi^{\prime}(0) \\
& +\sum_{j=1}^{i-1} \int_{(j-1) \tau}^{(j) \tau}(t-s)^{\gamma-1} E_{\gamma-\delta, \gamma}\left[A(t-s)^{\gamma-\delta}\right]\left[B \varphi_{j-1}(s-\tau)\right] d s
\end{aligned}
$$




$$
\begin{aligned}
& +\int_{(i-1) \tau}^{t}(t-s)^{\gamma-1} E_{\gamma-\delta, \gamma}\left[A(t-s)^{\gamma-\delta}\right]\left[B \varphi_{i-1}(s-\tau)\right] d s \\
& +\int_{0}^{t}(t-s)^{\gamma-1} E_{\gamma-\delta, \gamma}\left[A(t-s)^{\gamma-\delta}\right] C u(s) d s .
\end{aligned}
$$

Proof The conclusion obviously holds when $t \in[-\tau, 0]$. Denote $x(t)=\varphi(t)=\varphi_{0}(t), t \in$ $[-\tau, 0]$.

If $t \in\left[0, t_{1}\right]$, then from Lemma 2.5 we have

$$
\begin{aligned}
x(t)= & \varphi(0)+t E_{\gamma-\delta, 2}\left(A t^{\gamma-\delta}\right) \varphi^{\prime}(0) \\
& +\int_{0}^{t}(t-s)^{\gamma-1} E_{\gamma-\delta, \gamma}\left[A(t-s)^{\gamma-\delta}\right][B x(s-\tau)+C u(s)] d s, \\
x\left(t_{1}\right)= & \varphi(0)+t_{1} E_{\gamma-\delta, 2}\left(A t_{1}^{\gamma-\delta}\right) \varphi^{\prime}(0) \\
& +\int_{0}^{t_{1}}\left(t_{1}-s\right)^{\gamma-1} E_{\gamma-\delta, \gamma}\left[A\left(t_{1}-s\right)^{\gamma-\delta}\right]\left[B \varphi_{0}(s-\tau)+C u(s)\right] d s .
\end{aligned}
$$

If $t \in\left(t_{1}, \tau\right]$, then using [60], we have

$$
\begin{aligned}
x(t)= & x\left(t_{1}^{+}\right)-t_{1} E_{\gamma-\delta, 2}\left(A t_{1}^{\gamma-\delta}\right) \varphi^{\prime}(0) \\
& -\int_{0}^{t_{1}}\left(t_{1}-s\right)^{\gamma-1} E_{\gamma-\delta, \gamma}\left[A\left(t_{1}-s\right)^{\gamma-\delta}\right]\left[B \varphi_{0}(s-\tau)+C u(s)\right] d s \\
& +t E_{\gamma-\delta, 2}\left(A t^{\gamma-\delta}\right) \varphi^{\prime}(0)+\int_{0}^{t}(t-s)^{\gamma-1} E_{\gamma-\delta, \gamma}\left[A(t-s)^{\gamma-\delta}\right]\left[B \varphi_{0}(s-\tau)+C u(s)\right] d s, \\
x(t)= & x\left(t_{1}^{-}\right)+I_{1}\left(x\left(t_{1}^{-}\right)\right)-t_{1} E_{\gamma-\delta, 2}\left(A t_{1}^{\gamma-\delta}\right) \varphi^{\prime}(0) \\
& -\int_{0}^{t_{1}}\left(t_{1}-s\right)^{\gamma-1} E_{\gamma-\delta, \gamma}\left[A\left(t_{1}-s\right)^{\gamma-\delta}\right]\left[B \varphi_{0}(s-\tau)+C u(s)\right] d s \\
& +t E_{\gamma-\delta, 2}\left(A t^{\gamma-\delta}\right) \varphi^{\prime}(0)+\int_{0}^{t}(t-s)^{\gamma-1} E_{\gamma-\delta, \gamma}\left[A(t-s)^{\gamma-\delta}\right]\left[B \varphi_{0}(s-\tau)+C u(s)\right] d s, \\
x(t)= & \varphi(0)+I_{1}\left(x\left(t_{1}^{-}\right)\right)+t E_{\gamma-\delta, 2}\left(A t^{\gamma-\delta}\right) \varphi^{\prime}(0) \\
& +\int_{0}^{t}(t-s)^{\gamma-1} E_{\gamma-\delta, \gamma}\left[A(t-s)^{\gamma-\delta}\right]\left[B \varphi_{0}(s-\tau)+C u(s)\right] d s .
\end{aligned}
$$

Denote $\varphi_{1}(t)=x(t), t \in[0, \tau]$. If $t \in\left(\tau, t_{2}\right]$, then

$$
\begin{aligned}
x(t)= & x\left(t_{1}^{+}\right)-t_{1} E_{\gamma-\delta, 2}\left(A t_{1}^{\gamma-\delta}\right) \varphi^{\prime}(0) \\
& -\int_{0}^{t_{1}}\left(t_{1}-s\right)^{\gamma-1} E_{\gamma-\delta, \gamma}\left[A\left(t_{1}-s\right)^{\gamma-\delta}\right]\left[B \varphi_{0}(s-\tau)+C u(s)\right] d s \\
& +t E_{\gamma-\delta, 2}\left(A t^{\gamma-\delta}\right) \varphi^{\prime}(0)+\int_{0}^{\tau}(t-s)^{\gamma-1} E_{\gamma-\delta, \gamma}\left[A(t-s)^{\gamma-\delta}\right]\left[B \varphi_{0}(s-\tau)\right] d s \\
& +\int_{\tau}^{t}(t-s)^{\gamma-1} E_{\gamma-\delta, \gamma}\left[A(t-s)^{\gamma-\delta}\right]\left[B \varphi_{1}(s-\tau)\right] d s \\
& +\int_{0}^{t}(t-s)^{\gamma-1} E_{\gamma-\delta, \gamma}\left[A(t-s)^{\gamma-\delta}\right] C u(s) d s,
\end{aligned}
$$




$$
\begin{aligned}
x(t)= & x\left(t_{1}^{-}\right)+I_{1}\left(x\left(t_{1}^{-}\right)\right)-t_{1} E_{\gamma-\delta, 2}\left(A t_{1}^{\gamma-\delta}\right) \varphi^{\prime}(0) \\
& -\int_{0}^{t_{1}}\left(t_{1}-s\right)^{\gamma-1} E_{\gamma-\delta, \gamma}\left[A\left(t_{1}-s\right)^{\gamma-\delta}\right]\left[B \varphi_{0}(s-\tau)+C u(s)\right] d s \\
& +t E_{\gamma-\delta, 2}\left(A t^{\gamma-\delta}\right) \varphi^{\prime}(0)+\int_{0}^{\tau}(t-s)^{\gamma-1} E_{\gamma-\delta, \gamma}\left[A(t-s)^{\gamma-\delta}\right]\left[B \varphi_{0}(s-\tau)\right] d s \\
& +\int_{\tau}^{t}(t-s)^{\gamma-1} E_{\gamma-\delta, \gamma}\left[A(t-s)^{\gamma-\delta}\right]\left[B \varphi_{1}(s-\tau)\right] d s \\
& +\int_{0}^{t}(t-s)^{\gamma-1} E_{\gamma-\delta, \gamma}\left[A(t-s)^{\gamma-\delta}\right] C u(s) d s, \\
x(t)= & (0)+I_{1}\left(x\left(t_{1}^{-}\right)\right)+t E_{\gamma-\delta, 2}\left(A t^{\delta-\gamma}\right) \varphi^{\prime}(0) \\
& +\int_{0}^{\tau}(t-s)^{\delta-1} E_{\gamma-\delta, \gamma}\left[A(t-s)^{\gamma-\delta}\right] B \varphi_{0}(s-\tau) d s \\
& +\int_{\tau}^{t}(t-s)^{\gamma-1} E_{\gamma-\delta, \gamma}\left[A(t-s)^{\gamma-\delta}\right] B \varphi_{1}(s-\tau) d s \\
& +\int_{0}^{t}(t-s)^{\gamma-1} E_{\gamma-\delta, \gamma}\left[A(t-s)^{\gamma-\delta}\right] C u(s) d s .
\end{aligned}
$$

If $t \in\left(t_{2}, 2 \tau\right]$, then

$$
\begin{aligned}
& x(t)=x\left(t_{2}^{+}\right)-t_{2} E_{\gamma-\delta, 2}\left(A t_{2}^{\gamma-\delta}\right) \varphi^{\prime}(0) \\
& +\int_{0}^{\tau}\left(t_{2}-s\right)^{\gamma-1} E_{\gamma-\delta, \gamma}\left[A\left(t_{2}-s\right)^{\gamma-\delta}\right] B \varphi_{0}(s-\tau) d s \\
& +\int_{\tau}^{t_{2}}\left(t_{2}-s\right)^{\gamma-1} E_{\gamma-\delta, \gamma}\left[A\left(t_{2}-s\right)^{\gamma-\delta}\right] B \varphi_{1}(s-\tau) d s \\
& +\int_{0}^{t_{2}}\left(t_{2}-s\right)^{\gamma-1} E_{\gamma-\delta, \gamma}\left[A\left(t_{2}-s\right)^{\gamma-\delta}\right] C u(s) d s+t E_{\gamma-\delta, 2}\left(A t^{\gamma-\delta}\right) \varphi^{\prime}(0) \\
& +\int_{0}^{\tau}(t-s)^{\gamma-1} E_{\gamma-\delta, \gamma}\left[A(t-s)^{\gamma-\delta}\right] B \varphi_{0}(s-\tau) d s \\
& +\int_{\tau}^{t}(t-s)^{\gamma-1} E_{\gamma-\delta, \gamma}\left[A(t-s)^{\gamma-\delta}\right] B \varphi_{1}(s-\tau) d s \\
& +\int_{0}^{t}(t-s)^{\gamma-1} E_{\gamma-\delta, \gamma}\left[A(t-s)^{\gamma-\delta}\right] C u(s) d s, \\
& x(t)=x\left(t_{2}^{-}\right)+I_{2}\left(x\left(t_{2}^{-}\right)\right)-t_{2} E_{\gamma-\delta, 2}\left(A t_{2}^{\gamma-\delta}\right) \varphi^{\prime}(0) \\
& +\int_{0}^{\tau}\left(t_{2}-s\right)^{\gamma-1} E_{\gamma-\delta, \gamma}\left[A\left(t_{2}-s\right)^{\gamma-\delta}\right] B \varphi_{0}(s-\tau) d s \\
& +\int_{\tau}^{t_{2}}\left(t_{2}-s\right)^{\gamma-1} E_{\gamma-\delta, \gamma}\left[A\left(t_{2}-s\right)^{\gamma-\delta}\right] B \varphi_{1}(s-\tau) d s \\
& +\int_{0}^{t_{2}}\left(t_{2}-s\right)^{\gamma-1} E_{\gamma-\delta, \gamma}\left[A\left(t_{2}-s\right)^{\gamma-\delta}\right] C u(s) d s+t E_{\gamma-\delta, 2}\left(A t^{\gamma-\delta}\right) \varphi^{\prime}(0) \\
& +\int_{0}^{\tau}(t-s)^{\gamma-1} E_{\gamma-\delta, \gamma}\left[A(t-s)^{\gamma-\delta}\right] B \varphi_{0}(s-\tau) d s \\
& +\int_{\tau}^{t}(t-s)^{\gamma-1} E_{\gamma-\delta, \gamma}\left[A(t-s)^{\gamma-\delta}\right] B \varphi_{1}(s-\tau) d s \\
& +\int_{0}^{t}(t-s)^{\gamma-1} E_{\gamma-\delta, \gamma}\left[A(t-s)^{\gamma-\delta}\right] C u(s) d s,
\end{aligned}
$$




$$
\begin{aligned}
x(t)= & \varphi(0)+I_{1}\left(x\left(t_{1}^{-}\right)\right)+I_{2}\left(x\left(t_{2}^{-}\right)\right)+t E_{\gamma-\delta, 2}\left(A t^{\gamma-\delta}\right) \varphi^{\prime}(0) \\
& +\int_{0}^{\tau}(t-s)^{\gamma-1} E_{\gamma-\delta, \gamma}\left[A(t-s)^{\gamma-\delta}\right] B \varphi_{0}(s-\tau) d s \\
& +\int_{\tau}^{t}(t-s)^{\gamma-1} E_{\gamma-\delta, \gamma}\left[A(t-s)^{\gamma-\delta}\right] B \varphi_{1}(s-\tau) d s \\
& +\int_{0}^{t}(t-s)^{\gamma-1} E_{\gamma-\delta, \gamma}\left[A(t-s)^{\gamma-\delta}\right] C u(s) d s .
\end{aligned}
$$

Denote $x(t)=\varphi_{i}(t), t \in[(i-2) \tau,(i-1) \tau]$. If $t \in\left((i-1) \tau, t_{i}\right], i \geq 2$, then the same argument implies the following expression:

$$
\begin{aligned}
x(t)= & \varphi(0)+\sum_{j=1}^{i-1} I_{j}\left(x\left(t_{j}^{-}\right)\right)+t E_{\gamma-\delta, 2}\left(A t^{\gamma-\delta}\right) \varphi^{\prime}(0) \\
& +\sum_{j=1}^{i-1} \int_{(j-1) \tau}^{(j) \tau}(t-s)^{\gamma-1} E_{\gamma-\delta, \gamma}\left[A(t-s)^{\gamma-\delta}\right]\left[B \varphi_{j-1}(s-\tau)\right] d s \\
& +\int_{(i-1) \tau}^{t}(t-s)^{\gamma-1} E_{\gamma-\delta, \gamma}\left[A(t-s)^{\gamma-\delta}\right]\left[B \varphi_{i-1}(s-\tau)\right] d s \\
& +\int_{0}^{t}(t-s)^{\gamma-1} E_{\gamma-\delta, \gamma}\left[A(t-s)^{\gamma-\delta}\right] C u(s) d s .
\end{aligned}
$$

If $t \in\left(t_{i}, i \tau\right], i \geq 2$, then the same argument implies the following expression:

$$
\begin{aligned}
x(t)= & \varphi(0)+\sum_{j=1}^{i} I_{j}\left(x\left(t_{j}^{-}\right)\right)+t E_{\gamma-\delta, 2}\left(A t^{\gamma-\delta}\right) \varphi^{\prime}(0) \\
& +\sum_{j=1}^{i-1} \int_{(j-1) \tau}^{(j) \tau}(t-s)^{\gamma-1} E_{\gamma-\delta, \gamma}\left[A(t-s)^{\gamma-\delta}\right]\left[B \varphi_{j-1}(s-\tau)\right] d s \\
& +\int_{(i-1) \tau}^{t}(t-s)^{\gamma-1} E_{\gamma-\delta, \gamma}\left[A(t-s)^{\gamma-\delta}\right]\left[B \varphi_{i-1}(s-\tau)\right] d s \\
& +\int_{0}^{t}(t-s)^{\gamma-1} E_{\gamma-\delta, \gamma}\left[A(t-s)^{\gamma-\delta}\right] C u(s) d s .
\end{aligned}
$$

The proof is completed.

\section{Sufficient and necessary conditions for controllability of system}

In this part, we construct sufficient and necessary conditions for controllability criteria of system (1) by using the algebraic method.

Definition 3.1 For any initial function $\varphi \in C\left([-\tau, 0], \mathcal{R}^{n}\right)$ and any state $x_{\mu} \in \mathcal{R}^{n}$, system (1) on $[0, \mu](\mu \in(0, T])$ is called controllable if there exists a control input $u(t) \in$ $C_{p}\left([0, \mu], \mathcal{R}^{m}\right)$ such that the corresponding solution of (1) satisfies $x(\mu)=x_{\mu}$.

Theorem 3.2 System (1) is controllable on $[0, \mu]$ if and only if the Gramian matrix

$$
W_{c}[0, \mu]=\int_{0}^{\mu}(\mu-s)^{\gamma-1} E_{\gamma-\delta, \gamma}\left[A(\mu-s)^{\gamma-\delta}\right] C C^{*}\left[E_{\gamma-\delta, \gamma}\left(A^{*}(\mu-s)^{\gamma-\delta}\right)\right] d s
$$


is nonsingular for some $\mu \in[0, T]$, where $E_{\gamma-\delta, \gamma}$ is the Mittage-Leffler function, and $C^{*}$ is the transpose of a matrix $C$.

Proof First, we demonstrate the sufficiency. If $W_{c}[0, \mu]$ is nonsingular, then $W_{c}^{-1}[0, \mu]$ is well defined. For an initial state $\varphi \in C\left([-\tau, 0], \mathcal{R}^{n}\right)$, when $\mu \in\left[0, t_{1}\right]$, the control function can be taken as

$$
\begin{aligned}
u(t)= & C^{*}\left[E_{\gamma-\delta, \gamma}\left(A^{*}(\mu-s)^{\gamma-\delta}\right)\right] W_{c}^{-1}[0, \mu] \times\left[x_{\mu}-\varphi(0)-\mu E_{\gamma-\delta, 2}\left(A \mu^{\gamma-\delta}\right) \varphi^{\prime}(0)\right. \\
& \left.-\int_{0}^{\mu}(\mu-\theta)^{\gamma-1} E_{\gamma-\delta, \gamma}\left[A(\mu-\theta)^{\gamma-\delta}\right]\left[B \varphi_{0}(\theta-\tau) d \theta\right]\right]
\end{aligned}
$$

By changing $t=\mu$ in (10) and inserting (17) we have

$$
\begin{aligned}
x(\mu)= & \varphi(0)+\mu E_{\gamma-\delta, 2}\left(A \mu^{\gamma-\delta}\right) \varphi^{\prime}(0)+\int_{0}^{\mu}(\mu-s)^{\gamma-1} E_{\gamma-\delta, \gamma}\left[A(\mu-s)^{\gamma-\delta}\right] \\
& \times\left\{B \varphi_{0}(s-\tau)+C C^{*}\left[E_{\gamma-\delta, \gamma}\left(A^{*}(\mu-s)^{\gamma-\delta}\right)\right] W_{c}^{-1}[0, \mu]\right. \\
& \times\left[x_{\mu}-\varphi(0)-\mu E_{\gamma-\delta, 2}\left(A \mu^{\gamma-\delta}\right) \varphi^{\prime}(0)\right. \\
& \left.\left.-\int_{0}^{\mu}(\mu-\theta)^{\gamma-1} E_{\gamma-\delta, \gamma}\left[A(\mu-\theta)^{\gamma-\delta}\right]\left[B \varphi_{0}(\theta-\tau) d \theta\right]\right]\right\} d s, \\
x(\mu)= & \varphi(0)+\mu E_{\gamma-\delta, 2}\left(A \mu^{\gamma-\delta}\right) \varphi^{\prime}(0)+\int_{0}^{\mu}(\mu-s)^{\gamma-1} E_{\gamma-\delta, \gamma}\left[A(\mu-s)^{\gamma-\delta}\right] \\
& \times\left[B \varphi_{0}(s-\tau)\right] d s+x_{\mu}-\varphi(0)-\mu E_{\gamma-\delta, 2}\left(A \mu^{\gamma-\delta}\right) \varphi^{\prime}(0) \\
& -\int_{0}^{\mu}(\mu-\theta)^{\gamma-1} E_{\gamma-\delta, \gamma}\left[A(\mu-\theta)^{\gamma-\delta}\right]\left[B \varphi_{0}(\theta-\tau) d \theta\right],
\end{aligned}
$$

$x(\mu)=x_{\mu}$.

Thus system (1) is controllable on $[0, \mu], \mu \in\left[0, t_{1}\right]$.

For $\mu \in\left(t_{1}, \tau\right]$, the control function can be taken as

$$
\begin{aligned}
u(t)= & C^{*}\left[E_{\gamma-\delta, \gamma}\left(A^{*}(\mu-s)^{\gamma-\delta}\right)\right] W_{c}^{-1}[0, \mu] \\
& \times\left[x_{\mu}-\varphi(0)-I_{1}\left(x\left(t_{1}^{-}\right)\right)-\mu E_{\gamma-\delta, 2}\left(A \mu^{\gamma-\delta}\right) \varphi^{\prime}(0)\right. \\
& \left.-\int_{0}^{\mu}(\mu-\theta)^{\gamma-1} E_{\gamma-\delta, \gamma}\left[A(\mu-\theta)^{\gamma-\delta}\right]\left[B \varphi_{0}(\theta-\tau) d \theta\right]\right] .
\end{aligned}
$$

By changing $t=\mu$ in (11) and inserting (18) we have

$$
\begin{aligned}
x(\mu)= & \varphi(0)+I_{1}\left(x\left(t_{1}^{-}\right)\right)+\mu E_{\gamma-\delta, 2}\left(A \mu^{\gamma-\delta}\right) \varphi^{\prime}(0) \\
& +\int_{0}^{\mu}(\mu-s)^{\gamma-1} E_{\gamma-\delta, \gamma}\left[A(\mu-s)^{\gamma-\delta}\right] \times\left\{\left[B \varphi_{0}(s-\tau)\right.\right. \\
& +C C^{*}\left[E_{\gamma-\delta, \gamma}\left(A^{*}(\mu-s)^{\gamma-\delta}\right)\right] W_{c}^{-1}[0, \mu] \\
& \times\left[x_{\mu}-\varphi(0)-I_{1}\left(x\left(t_{1}^{-}\right)\right)-\mu E_{\gamma-\delta, 2}\left(A \mu^{\gamma-\delta}\right) \varphi^{\prime}(0)\right]
\end{aligned}
$$




$$
\begin{aligned}
& \left.-\int_{0}^{\mu}(\mu-\theta)^{\gamma-1} E_{\gamma-\delta, \gamma}\left[A(\mu-\theta)^{\gamma-\delta}\right]\left[B \varphi_{0}(\theta-\tau) d \theta\right]\right\} d s, \\
x(\mu)= & \varphi(0)+I_{1}\left(x\left(t_{1}^{-}\right)\right)+\mu E_{\gamma-\delta, 2}\left(A \mu^{\gamma-\delta}\right) \varphi^{\prime}(0) \\
& +\int_{0}^{\mu}(\mu-s)^{\gamma-1} E_{\gamma-\delta, \gamma}\left[A(\mu-s)^{\gamma-\delta}\right] \times\left[B \varphi_{0}(s-\tau)\right] d s \\
& +x_{\mu}-\varphi(0)-I_{1}\left(x\left(t_{1}^{-}\right)\right)-\mu E_{\gamma-\delta, 2}\left(A \mu^{\gamma-\delta}\right) \varphi^{\prime}(0) \\
& -\int_{0}^{\mu}(\mu-\theta)^{\gamma-1} E_{\gamma-\delta, \gamma}\left[A(\mu-\theta)^{\gamma-\delta}\right]\left[B \varphi_{0}(\theta-\tau) d \theta\right],
\end{aligned}
$$

$x(\mu)=x_{\mu}$.

Hence the system is controllable on $[0, \mu], \mu \in\left[t_{1}, \tau\right]$.

For $\mu \in\left(\tau, t_{2}\right]$, the control function can be taken as

$$
\begin{aligned}
u(t)= & C^{*}\left[E_{\gamma-\delta, \gamma}\left(A^{*}(\mu-s)^{\gamma-\delta}\right)\right] W_{c}^{-1}[0, \mu] \\
& \times\left[x_{\mu}-\varphi(0)-I_{1}\left(x\left(t_{1}^{-}\right)\right)-\mu E_{\gamma-\delta, 2}\left(A \mu^{\gamma-\delta}\right) \varphi^{\prime}(0)\right] \\
& -\int_{0}^{\tau}(\mu-\theta)^{\gamma-1} E_{\gamma-\delta, \gamma}\left[A(\mu-\theta)^{\gamma-\delta}\right] B \varphi_{0}(\theta-\tau) d \theta \\
& -\int_{\tau}^{\mu}(\mu-\theta)^{\gamma-1} E_{\gamma-\delta, \gamma}\left[A(\mu-\theta)^{\gamma-\delta}\right] B \varphi_{1}(\theta-\tau) d \theta .
\end{aligned}
$$

By changing $t=\mu$ in (12) and inserting (19) we have

$$
\begin{aligned}
x(\mu)= & \varphi(0)+I_{1}\left(x\left(t_{1}^{-}\right)\right)+\mu E_{\gamma-\delta, 2}\left(A \mu^{\gamma-\delta}\right) \varphi^{\prime}(0) \\
& +\int_{0}^{\tau}(\mu-s)^{\gamma-1} E_{\gamma-\delta, \gamma}\left[A(\mu-s)^{\gamma-\delta}\right] B \varphi_{0}(s-\tau) d s \\
& +\int_{\tau}^{\mu}(\mu-s)^{\gamma-1} E_{\gamma-\delta, \gamma}\left[A(\mu-s)^{\gamma-\delta}\right] B \varphi_{1}(s-\tau) d s \\
& +\int_{0}^{\mu}(\mu-s)^{\gamma-1} E_{\gamma-\delta, \gamma}\left[A(\mu-s)^{\gamma-\delta}\right]\left\{C C^{*}\left[E_{\gamma-\delta, \gamma}\left(A^{*}(\mu-s)^{\gamma-\delta}\right)\right] W_{c}^{-1}[0, \mu]\right. \\
& \times x_{\mu}-\varphi(0)-I_{1}\left(x\left(t_{1}^{-}\right)\right)-\mu E_{\gamma-\delta, 2}\left(A \mu^{\gamma-\delta}\right) \varphi^{\prime}(0) \\
& -\int_{0}^{\tau}(\mu-\theta)^{\gamma-1} E_{\gamma-\delta, \gamma}\left[A(\mu-\theta)^{\gamma-\delta}\right] B \varphi_{0}(\theta-\tau) d \theta \\
& \left.-\int_{\tau}^{\mu}(\mu-\theta)^{\gamma-1} E_{\gamma-\delta, \gamma}\left[A(\mu-\theta)^{\gamma-\delta}\right] B \varphi_{1}(\theta-\tau) d \theta\right\} d s, \\
x(\mu)= & \varphi(0)+I_{1}\left(x\left(t_{1}^{-}\right)\right)+\mu E_{\gamma-\delta, 2}\left(A \mu^{\gamma-\delta}\right) \varphi^{\prime}(0) \\
& +\int_{0}^{\tau}(\mu-s)^{\gamma-1} E_{\gamma-\delta, \gamma}\left[A(\mu-s)^{\gamma-\delta}\right] B \varphi_{0}(s-\tau) d s \\
& +\int_{\tau}^{\mu}(\mu-s)^{\gamma-1} E_{\gamma-\delta, \gamma}\left[A(\mu-s)^{\gamma-\delta}\right] B \varphi_{1}(s-\tau) d s \\
& +x_{\mu}-\varphi(0)-I_{1}\left(x\left(t_{1}^{-}\right)\right)-\mu E_{\gamma-\delta, 2}\left(A \mu^{\gamma-\delta}\right) \varphi^{\prime}(0)
\end{aligned}
$$




$$
\begin{aligned}
& -\int_{0}^{\tau}(\mu-\theta)^{\gamma-1} E_{\gamma-\delta, \gamma}\left[A(\mu-\theta)^{\gamma-\delta}\right] B \varphi_{0}(\theta-\tau) d \theta \\
& -\int_{\tau}^{\mu}(\mu-\theta)^{\gamma-1} E_{\gamma-\delta, \gamma}\left[A(\mu-\theta)^{\gamma-\delta}\right] B \varphi_{1}(\theta-\tau) d \theta,
\end{aligned}
$$

$x(\mu)=x_{\mu}$.

Hence system (1) is controllable on $[0, \mu], \mu \in\left[t_{1}, \tau\right]$.

For $\mu \in\left(t_{2}, 2 \tau\right]$, the control function can be taken as

$$
\begin{aligned}
u(t)= & C^{*}\left[E_{\gamma-\delta, \gamma}\left(A^{*}(\mu-s)^{\gamma-\delta}\right)\right] W_{c}^{-1}[0, \mu] \times\left[x_{\mu}-\varphi(0)-I_{1}\left(x\left(t_{1}^{-}\right)\right)-I_{2}\left(x\left(t_{2}^{-}\right)\right)\right. \\
& -\mu E_{\gamma-\delta, 2}\left(A \mu^{\gamma-\delta}\right) \varphi^{\prime}(0)-\int_{0}^{\tau}(\mu-\theta)^{\gamma-1} E_{\gamma-\delta, \gamma}\left[A(\mu-\theta)^{\gamma-\delta}\right] B \varphi_{0}(\theta-\tau) d \theta \\
& -\int_{\tau}^{\mu}(\mu-\theta)^{\gamma-1} E_{\gamma-\delta, \gamma}\left[A(\mu-\theta)^{\gamma-\delta}\right] B \varphi_{1}(\theta-\tau) d \theta .
\end{aligned}
$$

By changing $t=\mu$ in (13) and inserting (20) we have

$$
\begin{aligned}
x(\mu)= & \varphi(0)+I_{1}\left(x\left(t_{1}^{-}\right)\right)+I_{2}\left(x\left(t_{2}^{-}\right)\right)+\mu E_{\gamma-\delta, 2}\left(A \mu^{\gamma-\delta}\right) \varphi^{\prime}(0) \\
& +\int_{0}^{\tau}(\mu-s)^{\gamma-1} E_{\gamma-\delta, \gamma}\left[A(\mu-s)^{\gamma-\delta}\right] B \varphi_{0}(s-\tau) d s \\
& +\int_{\tau}^{\mu}(\mu-s)^{\gamma-1} E_{\gamma-\delta, \gamma}\left[A(\mu-s)^{\gamma-\delta}\right] B \varphi_{1}(s-\tau) d s \\
& +\int_{0}^{\mu}(\mu-s)^{\gamma-1} E_{\gamma-\delta, \gamma}\left[A(\mu-s)^{\gamma-\delta}\right]\left\{C C^{*}\left[E_{\gamma-\delta, \gamma}\left(A^{*}(\mu-s)^{\gamma-\delta}\right)\right] W_{c}^{-1}[0, \mu]\right. \\
& \times\left[x_{\mu}-\varphi(0)-I_{1}\left(x\left(t_{1}^{-}\right)\right)-I_{2}\left(x\left(t_{2}^{-}\right)\right)-\mu E_{\gamma-\delta, 2}\left(A \mu^{\gamma-\delta}\right) \varphi^{\prime}(0)\right. \\
& -\int_{0}^{\tau}(\mu-\theta)^{\gamma-1} E_{\gamma-\delta, \gamma}\left[A(\mu-\theta)^{\gamma-\delta}\right] B \varphi_{0}(\theta-\tau) d \theta \\
& \left.-\int_{\tau}^{\mu}(\mu-\theta)^{\gamma-1} E_{\gamma-\delta, \gamma}\left[A(\mu-\theta)^{\gamma-\delta}\right] B \varphi_{1}(\theta-\tau) d \theta\right] d s, \\
x(\mu)= & \varphi(0)+I_{1}\left(x\left(t_{1}^{-}\right)\right)+I_{2}\left(x\left(t_{2}^{-}\right)\right)+\mu E_{\gamma-\delta, 2}\left(A \mu^{\gamma-\delta}\right) \varphi^{\prime}(0) \\
& +\int_{0}^{\tau}(\mu-s)^{\gamma-1} E_{\gamma-\delta, \gamma}\left[A(\mu-s)^{\gamma-\delta}\right] B \varphi_{0}(s-\tau) d s \\
& +\int_{\tau}^{\mu}(\mu-s)^{\gamma-1} E_{\gamma-\delta, \gamma}\left[A(\mu-s)^{\gamma-\delta}\right] B \varphi_{1}(s-\tau) d s \\
& +x_{\mu}-\varphi(0)-I_{1}\left(x\left(t_{1}^{-}\right)\right)-I_{2}\left(x\left(t_{2}^{-}\right)\right)-\mu E_{\gamma-\delta, 2}\left(A \mu^{\gamma-\delta}\right) \varphi^{\prime}(0) \\
& -\int_{0}^{\tau}(\mu-\theta)^{\gamma-1} E_{\gamma-\delta, \gamma}\left[A(\mu-\theta)^{\gamma-\delta}\right] B \varphi_{0}(\theta-\tau) d \theta \\
& -\int_{\tau}^{\mu}(\mu-\theta)^{\gamma-1} E_{\gamma-\delta, \gamma}\left[A(\mu-\theta)^{\gamma-\delta}\right] B \varphi_{1}(\theta-\tau) d \theta,
\end{aligned}
$$

$x(\mu)=x_{\mu}$.

Hence system (1) is controllable on $[0, \mu], \mu \in\left(t_{2}, 2 \tau\right]$. 
For $\mu \in\left((i-1) \tau, t_{i}\right], i \geq 2$, the control function can be taken as

$$
\begin{aligned}
u(t)= & C^{*}\left[E_{\gamma-\delta, \gamma}\left(A^{*}(\mu-s)^{\gamma-\delta}\right)\right] W_{c}^{-1}[0, \mu] \\
& \times\left[x_{\mu}-\varphi(0)-\sum_{j=1}^{i-1} I_{j}\left(x\left(t_{j}^{-}\right)\right)-\mu E_{\gamma-\delta, 2}\left(A \mu^{\gamma-\delta}\right) \varphi^{\prime}(0)\right] \\
& -\sum_{j=1}^{i-1} \int_{(j-1) \tau}^{(j) \tau}(\mu-\theta)^{\gamma-1} E_{\gamma-\delta, \gamma}\left[A(\mu-\theta)^{\gamma-\delta}\right]\left[B \varphi_{j-1}(\theta-\tau)\right] d \theta \\
& -\int_{(i-1) \tau}^{\mu}(\mu-\theta)^{\gamma-1} E_{\gamma-\delta, \gamma}\left[A(\mu-\theta)^{\gamma-\delta}\right]\left[B \varphi_{i-1}(\theta-\tau)\right] d \theta .
\end{aligned}
$$

Substituting $t=\mu$ into (14) and inserting (21) we have

$$
\begin{aligned}
& x(\mu)=\varphi(0)+\sum_{j=1}^{i-1} I_{j}\left(x\left(t_{j}^{-}\right)\right)+\mu E_{\gamma-\delta, 2}\left(A \mu^{\gamma-\delta}\right) \varphi^{\prime}(0) \\
& +\sum_{j=1}^{i-1} \int_{(j-1) \tau}^{(j) \tau}(\mu-s)^{\gamma-1} E_{\gamma-\delta, \gamma}\left[A(\mu-s)^{\gamma-\delta}\right]\left[B \varphi_{j-1}(s-\tau)\right] d s \\
& +\int_{(i-1) \tau}^{\mu}(\mu-s)^{\gamma-1} E_{\gamma-\delta, \gamma}\left[A(\mu-s)^{\gamma-\delta}\right]\left[B \varphi_{i-1}(s-\tau)\right] d s \\
& +\int_{0}^{\mu}(\mu-s)^{\gamma-1} E_{\gamma-\delta, \gamma}\left[A(\mu-s)^{\gamma-\delta}\right]\left\{C C^{*}\left[E_{\gamma-\delta, \gamma}\left(A^{*}(\mu-s)^{\gamma-\delta}\right)\right] W_{c}^{-1}[0, \mu]\right. \\
& \times\left[x_{\mu}-\varphi(0)-\sum_{j=1}^{i-1} I_{j}\left(x\left(t_{j}^{-}\right)\right)-\mu E_{\gamma-\delta, 2}\left(A \mu^{\gamma-\delta}\right) \varphi^{\prime}(0)\right. \\
& -\sum_{j=1}^{i-1} \int_{(j-1) \tau}^{(j) \tau}(\mu-\theta)^{\gamma-1} E_{\gamma-\delta, \gamma}\left[A(\mu-\theta)^{\gamma-\delta}\right]\left[B \varphi_{j-1}(\theta-\tau)\right] d \theta \\
& \left.-\int_{(i-1) \tau}^{\mu}(\mu-\theta)^{\gamma-1} E_{\gamma-\delta, \gamma}\left[A(\mu-\theta)^{\gamma-\delta}\right]\left[B \varphi_{i-1}(\theta-\tau)\right] d \theta\right] d s, \\
& x(\mu)=\varphi(0)+\sum_{j=1}^{i-1} I_{j}\left(x\left(t_{j}^{-}\right)\right)+\mu E_{\gamma-\delta, 2}\left(A \mu^{\gamma-\delta}\right) \varphi^{\prime}(0) \\
& +\sum_{j=1}^{i-1} \int_{(j-1) \tau}^{(j) \tau}(\mu-s)^{\gamma-1} E_{\gamma-\delta, \gamma}\left[A(\mu-s)^{\gamma-\delta}\right]\left[B \varphi_{j-1}(s-\tau)\right] d s \\
& +\int_{(i-1) \tau}^{\mu}(\mu-s)^{\gamma-1} E_{\gamma-\delta, \gamma}\left[A(\mu-s)^{\gamma-\delta}\right]\left[B \varphi_{i-1}(s-\tau)\right] d s \\
& +x_{\mu}-\varphi(0)-\sum_{j=1}^{i-1} I_{j}\left(x\left(t_{j}^{-}\right)\right)-\mu E_{\gamma-\delta, 2}\left(A \mu^{\gamma-\delta}\right) \varphi^{\prime}(0) \\
& -\sum_{j=1}^{i-1} \int_{(j-1) \tau}^{(j) \tau}(\mu-\theta)^{\gamma-1} E_{\gamma-\delta, \gamma}\left[A(\mu-\theta)^{\gamma-\delta}\right]\left[B \varphi_{j-1}(\theta-\tau)\right] d \theta \\
& -\int_{(i-1) \tau}^{\mu}(\mu-\theta)^{\gamma-1} E_{\gamma-\delta, \gamma}\left[A(\mu-\theta)^{\gamma-\delta}\right]\left[B \varphi_{i-1}(\theta-\tau)\right] d \theta,
\end{aligned}
$$

$x(\mu)=x_{\mu}$. 
Hence system (1) is controllable on $[0, \mu], \mu \in\left((k-1) \tau, t_{k}\right]$.

For $\mu \in\left(t_{i}, i \tau\right], i \geq 2$, we have the control function

$$
\begin{aligned}
u(t)= & C^{*}\left[E_{\gamma-\delta, \gamma}\left(A^{*}(\mu-s)^{\gamma-\delta}\right)\right] W_{c}^{-1}[0, \mu] \\
& \times\left[x_{\mu}-\varphi(0)-\sum_{j=1}^{i} I_{j}\left(x\left(t_{j}^{-}\right)\right)-\mu E_{\gamma-\delta, 2}\left(A \mu^{\gamma-\delta}\right) \varphi^{\prime}(0)\right] \\
& -\sum_{j=1}^{i-1} \int_{(j-1) \tau}^{(j) \tau}(\mu-\theta)^{\gamma-1} E_{\gamma-\delta, \gamma}\left[A(\mu-\theta)^{\gamma-\delta}\right]\left[B \varphi_{j-1}(\theta-\tau)\right] d \theta \\
& -\int_{(i-1) \tau}^{\mu}(\mu-\theta)^{\gamma-1} E_{\gamma-\delta, \gamma}\left[A(\mu-\theta)^{\gamma-\delta}\right]\left[B \varphi_{i-1}(\theta-\tau)\right] d \theta .
\end{aligned}
$$

By changing $t=\mu$ in (15) and inserting (22) we have

$$
\begin{aligned}
& x(\mu)=\varphi(0)+\sum_{j=1}^{i} I_{j}\left(x\left(t_{j}^{-}\right)\right)+\mu E_{\gamma-\delta, 2}\left(A \mu^{\gamma-\delta}\right) \varphi^{\prime}(0) \\
& +\sum_{j=1}^{i-1} \int_{(j-1) \tau}^{(j) \tau}(\mu-s)^{\gamma-1} E_{\gamma-\delta, \gamma}\left[A(\mu-s)^{\gamma-\delta}\right]\left[B \varphi_{j-1}(s-\tau)\right] d s \\
& +\int_{(i-1) \tau}^{\mu}(\mu-s)^{\gamma-1} E_{\gamma-\delta, \gamma}\left[A(\mu-s)^{\gamma-\delta}\right]\left[B \varphi_{i-1}(s-\tau)\right] d s \\
& +\int_{0}^{\mu}(\mu-s)^{\gamma-1} E_{\gamma-\delta, \gamma}\left[A(\mu-s)^{\gamma-\delta}\right] C C^{*}\left[E_{\gamma-\delta, \gamma}\left(A^{*}(\mu-s)^{\gamma-\delta}\right)\right] W_{c}^{-1}[0, \mu] \\
& \times\left[x_{\mu}-\varphi(0)-\sum_{j=1}^{i} I_{j}\left(x\left(t_{j}^{-}\right)\right)-\mu E_{\gamma-\delta, 2}\left(A \mu^{\gamma-\delta}\right) \varphi^{\prime}(0)\right. \\
& -\sum_{j=1}^{i-1} \int_{(j-1) \tau}^{(j) \tau}(\mu-\theta)^{\gamma-1} E_{\gamma-\delta, \gamma}\left[A(\mu-\theta)^{\gamma-\delta}\right]\left[B \varphi_{j-1}(\theta-\tau)\right] d \theta \\
& \left.-\int_{(i-1) \tau}^{\mu}(\mu-\theta)^{\gamma-1} E_{\gamma-\delta, \gamma}\left[A(\mu-\theta)^{\gamma-\delta}\right]\left[B \varphi_{i-1}(\theta-\tau)\right] d \theta\right] d s, \\
& x(\mu)=\varphi(0)+\sum_{j=1}^{i} I_{j}\left(x\left(t_{j}^{-}\right)\right)+\mu E_{\gamma-\delta, 2}\left(A \mu^{\gamma-\delta}\right) \varphi^{\prime}(0) \\
& +\sum_{j=1}^{i-1} \int_{(j-1) \tau}^{(j) \tau}(\mu-s)^{\gamma-1} E_{\gamma-\delta, \gamma}\left[A(\mu-s)^{\gamma-\delta}\right]\left[B \varphi_{j-1}(s-\tau)\right] d s \\
& +\int_{(i-1) \tau}^{\mu}(\mu-s)^{\gamma-1} E_{\gamma-\delta, \gamma}\left[A(\mu-s)^{\gamma-\delta}\right]\left[B \varphi_{i-1}(s-\tau)\right] d s \\
& +x_{\mu}-\varphi(0)-\sum_{j=1}^{i} I_{j}\left(x\left(t_{j}^{-}\right)\right)-\mu E_{\gamma-\delta, 2}\left(A \mu^{\gamma-\delta}\right) \varphi^{\prime}(0) \\
& -\sum_{j=1}^{i-1} \int_{(j-1) \tau}^{(j) \tau}(\mu-\theta)^{\gamma-1} E_{\gamma-\delta, \gamma}\left[A(\mu-\theta)^{\gamma-\delta}\right]\left[B \varphi_{j-1}(\theta-\tau)\right] d \theta
\end{aligned}
$$




$$
-\int_{(i-1) \tau}^{\mu}(\mu-\theta)^{\gamma-1} E_{\gamma-\delta, \gamma}\left[A(\mu-\theta)^{\gamma-\delta}\right]\left[B \varphi_{i-1}(\theta-\tau)\right] d \theta
$$

$x(\mu)=x_{\mu}$.

Hence system (1) is controllable on $[0, \mu]$. Furthermore, we prove the necessity of Theorem 3.2. Suppose, without loss of generality, that $W_{c}[0, \mu]$ is singular. For $\mu \in\left((i-1) \tau, t_{i}\right]$, $i \geq 2$, there exists a nonzero vector $z_{0}$ such that

$$
z_{0}^{*} W_{c}[0, \mu] z_{0}=0,
$$

that is,

$$
\int_{0}^{\mu} z_{0}^{*}(\mu-s)^{\gamma-1} E_{\gamma-\delta, \gamma}\left[A(\mu-s)^{\gamma-\delta}\right] C C^{*}\left[E_{\gamma-\delta, \gamma}\left(A^{*}(\mu-s)^{\gamma-\delta}\right)\right] z_{0} d s=0 .
$$

Then it follows that

$$
z_{0}^{*} E_{\gamma-\delta, \gamma}\left[A(\mu-s)^{\gamma-\delta}\right] C=0
$$

on $s \in[0, \mu]$. Since system (1) is controllable, there exist control inputs $u_{1}(t)$ and $u_{2}(t)$ such that

$$
\begin{aligned}
x(\mu)= & \varphi(0)+\sum_{j=1}^{i-1} I_{j}\left(x\left(t_{j}^{-}\right)\right)+\mu E_{\gamma-\delta, 2}\left(A \mu^{\gamma-\delta}\right) \varphi^{\prime}(0) \\
& +\sum_{j=1}^{i-1} \int_{(j-1) \tau}^{(j) \tau}(\mu-s)^{\gamma-1} E_{\gamma-\delta, \gamma}\left[A(\mu-s)^{\gamma-\delta}\right]\left[B \varphi_{j-1}(s-\tau)\right] d s \\
& +\int_{(i-1) \tau}^{\mu}(\mu-s)^{\gamma-1} E_{\gamma-\delta, \gamma}\left[A(\mu-s)^{\gamma-\delta}\right]\left[B \varphi_{i-1}(s-\tau)\right] d s \\
& +\int_{0}^{\mu}(\mu-s)^{\gamma-1} E_{\gamma-\delta, \gamma}\left[A(\mu-s)^{\gamma-\delta}\right] C u_{1}(s) d s=0, \\
z_{0}= & (0)+\sum_{j=1}^{i-1} I_{j}\left(x\left(t_{j}^{-}\right)\right)+\mu E_{\gamma-\delta, 2}\left(A \mu^{\gamma-\delta}\right) \varphi^{\prime}(0) \\
& +\sum_{j=1}^{i-1} \int_{(j-1) \tau}^{(j) \tau}(\mu-s)^{\gamma-1} E_{\gamma-\delta, \gamma}\left[A(\mu-s)^{\gamma-\delta}\right]\left[B \varphi_{j-1}(s-\tau)\right] d s \\
& +\int_{(i-1) \tau}^{\mu}(\mu-s)^{\gamma-1} E_{\gamma-\delta, \gamma}\left[A(\mu-s)^{\gamma-\delta}\right]\left[B \varphi_{i-1}(s-\tau)\right] d s \\
& +\int_{0}^{\mu}(\mu-s)^{\gamma-1} E_{\gamma-\delta, \gamma}\left[A(\mu-s)^{\gamma-\delta}\right] C u_{2}(s) d s .
\end{aligned}
$$

Combining (26) and (27) yields

$$
z_{0}-\int_{0}^{\mu}(\mu-s)^{\gamma-1} E_{\gamma-\delta, \gamma}\left[A(\mu-s)^{\gamma-\delta}\right] C\left[u_{2}(s)-u_{1}(s)\right] d s=0 .
$$


Multiplying both sides of (28) by $z_{0}^{*}$ we have

$$
z_{0}^{*} z_{0}-\int_{0}^{\mu}(\mu-s)^{\gamma-1} z_{0}^{*} E_{\gamma-\delta, \gamma}\left[A(\mu-s)^{\gamma-\delta}\right] \times C\left[u_{2}(s)-u_{1}(s)\right] d s=0 .
$$

Since $z_{0}^{*} E_{\gamma-\delta, \gamma}\left[A(\mu-s)^{\gamma-\delta}\right] C=0$, we have $z_{0}^{*} z_{0}=0$. Thus $z_{0}=0$. This contradiction completes the proof sufficiency.

Next, we establish the necessity. Suppose, without loss of generality, that $W_{c}[0, \mu]$ is singular. For $t \in\left(t_{i}, i \tau\right], i \geq 2$, the proof is similar, so we omit it. This completes the proof of the theorem.

Theorem 3.3 System (1) on $[0, \mu]$ is controllable if and only if $\operatorname{rank}\left[C|A C| \cdots \mid A^{n-1} C\right]=n$.

Proof According to the theorem of Cayley-Hamilton, $t E_{\gamma-\delta, 2}\left(A t^{\gamma-\delta}\right), t^{\gamma-1} E_{\gamma-\delta, \gamma}\left(A t^{\gamma-\delta}\right)$ can be represented as

$$
\begin{aligned}
& t E_{\gamma-\delta, 2}\left(A t^{\gamma-\delta}\right)=\sum_{i=0}^{\infty} \frac{t^{i}(\gamma-\delta)+1}{\Gamma(i(\gamma-\delta)+2)} A^{i}=\sum_{i=0}^{n-1} G_{1 i}(t) A^{i}, \\
& t^{\gamma-1} E_{\gamma-\delta, \gamma}\left(A t^{\gamma-\delta}\right)=\sum_{i=0}^{\infty} \frac{t^{i}(\gamma-\delta)+\gamma-1}{\Gamma(i(\gamma-\delta)+\gamma)} A^{i}=\sum_{i=0}^{n-1} G_{2 i}(t) A^{i} .
\end{aligned}
$$

For $\mu \in\left[0, t_{1}\right]$, we have

$$
\begin{aligned}
x(\mu)= & \varphi(0)+\mu E_{\gamma-\delta, 2}\left(A \mu^{\gamma-\delta}\right) \varphi^{\prime}(0) \\
& +\int_{0}^{\mu}(\mu-s)^{\gamma-1} E_{\gamma-\delta, \gamma}\left[A(\mu-s)^{\gamma-\delta}\right]\left[B \varphi_{0}(s-\tau)+C u(s)\right] d s, \\
x(\mu)= & \varphi(0)+\sum_{i=0}^{n-1} G_{1 i} \mu A^{i} \varphi^{\prime}(0) \\
& +\sum_{i=0}^{n-1} \int_{0}^{\mu} G_{2 i}(\mu-s) A^{i}\left[B \varphi_{0}(s-\tau)+C u(s)\right] d s .
\end{aligned}
$$

Let

$$
\psi=\varphi(0)+\sum_{i=0}^{n-1} G_{1 i} \mu A^{i} \varphi^{\prime}(0)+\sum_{i=0}^{n-1} \int_{0}^{\mu} G_{2 i}(\mu-s) A^{i}\left[B \varphi_{0}(s-\tau)\right] d s
$$

Combining (33) with (34) we have

$$
\begin{aligned}
x(\mu)-\psi & =\sum_{i=0}^{n-1} A^{i} C \int_{0}^{\mu} G_{2 i}(\mu-s) u(s) d s \\
& =\left[C|A C| \cdots \mid A^{n-1} C\right]\left(\begin{array}{c}
d_{0} \\
d_{1} \\
\cdot \\
\cdot \\
\cdot \\
d_{n-1}
\end{array}\right),
\end{aligned}
$$


where $d_{i}=\int_{0}^{\mu} G_{2 i}(t)(\mu-s) u(s) d s, i=0,1,2, \ldots, n-1$. For arbitrary $\varphi \in C\left([-\tau, 0], \mathcal{R}^{n}\right)$, sufficient and necessary condition to have a control input $u(t)$ satisfying (36) is

$$
\operatorname{rank}\left[C|A C| \cdots \mid A^{n-1} C\right]=n \text {. }
$$

For $\mu \in\left((i-1) \tau, t_{i}\right]$, we have

$$
\begin{aligned}
x(\mu)= & \varphi(0)+\sum_{j=1}^{i-1} I_{j}\left(x\left(t_{j}^{-}\right)\right)+\mu E_{\gamma-\delta, 2}\left(A \mu^{\gamma-\delta}\right) \varphi^{\prime}(0) \\
& +\sum_{j=1}^{i-1} \int_{(j-1) \tau}^{(j) \tau}(\mu-s)^{\gamma-1} E_{\gamma-\delta, \gamma}\left[A(\mu-s)^{\gamma-\delta}\right]\left[B \varphi_{j-1}(s-\tau)\right] d s \\
& +\int_{(i-1) \tau}^{\mu}(\mu-s)^{\gamma-1} E_{\gamma-\delta, \gamma}\left[A(\mu-s)^{\gamma-\delta}\right]\left[B \varphi_{i-1}(s-\tau)\right] d s \\
& +\int_{0}^{\mu}(\mu-s)^{\gamma-1} E_{\gamma-\delta, \gamma}\left[A(t-s)^{\gamma-\delta}\right] C u(s) d s, \\
x(\mu)= & \varphi(0)+\sum_{j=1}^{i-1} I_{j}\left(x\left(t_{j}^{-}\right)\right)+\sum_{i=0}^{n-1} G_{1 i} \mu A^{i} \varphi^{\prime}(0) \\
& +\sum_{j=1}^{i-1} \sum_{i=0}^{n-1} \int_{(j-1) \tau}^{(j) \tau} G_{2 i}(\mu-s) A^{i}\left[B \varphi_{j-1}(s-\tau)\right] d s \\
& +\sum_{i=0}^{n-1} \int_{(i-1) \tau}^{\mu} G_{2 i}(\mu-s) A^{i}\left[B \varphi_{i-1}(s-\tau)\right] d s \\
& +\sum_{i=0}^{n-1} A^{i} C \int_{0}^{\mu} G_{2 i}(\mu-s) u(s) d s, \\
\psi_{1}= & (0)+\sum_{j=1}^{i-1} I_{j}\left(x\left(t_{j}^{-}\right)\right)+\sum_{i=0}^{n-1} G_{1 i} \mu A^{i} \varphi^{\prime}(0) \\
+ & \sum_{j=1}^{i-1} \sum_{i=0}^{n-1} \int_{(j-1) \tau}^{(j) \tau} G_{2 i}(\mu-s) A^{i}\left[B \varphi_{j-1}(s-\tau)\right] d s \\
& G_{2 i}(\mu-s) A^{i}\left[B \varphi_{i-1}(s-\tau)\right] d s . \\
&
\end{aligned}
$$

Combining (37) with (38) we have

$$
\begin{aligned}
x(\mu)-\psi_{1} & =\sum_{i=0}^{n-1} A^{i} C \int_{0}^{\mu} G_{2 i}(\mu-s) u(s) d s \\
& =\left[C|A C| \cdots \mid A^{n-1} C\right]\left(\begin{array}{c}
d_{0} \\
d_{1} \\
\cdot \\
\cdot \\
\cdot \\
d_{n-1}
\end{array}\right) .
\end{aligned}
$$


For arbitrary $\varphi \in C\left([-\tau, 0], \mathcal{R}^{n}\right)$, a sufficient and necessary condition to have a control input $u(t)$ satisfying (39) is that

$$
\operatorname{rank}\left[C|A C| \cdots \mid A^{n-1} C\right]=n \text {. }
$$

For $t \in\left(t_{i}, i \tau\right], i \geq 2$, the proof is similar, and we omit it. Thus the proof is completed.

Remark 3.4 System (1) can be controlled only in cases where the resolvent condition $\lambda\left(\lambda I+Q_{w}\right)^{-1} \rightarrow 0$ as $\lambda \rightarrow 0$ holds (here $Q_{w}$ is the respective Gramian matrix in the nonfractional, nondelay, and nonimpulsive case) since this is equivalent to the rank condition in the finite-dimensional case $[24,61]$.

\section{Example}

In this section, we apply the results for the controllability criterion we acquired in the past section.

Example 1 Consider the controllability of the following damped fractional differential system with state delay and impulses:

$$
\begin{aligned}
& { }^{c} D^{3 / 2} x(t)-\left(\begin{array}{ll}
0 & 1 \\
0 & 0
\end{array}\right){ }^{c} D^{1 / 2} x(t) \\
& =\left(\begin{array}{cr}
-1 & 2 \\
1 & 3
\end{array}\right) x\left(t-\frac{1}{4}\right)+\left(\begin{array}{l}
1 \\
2
\end{array}\right) u(t), \quad t \in[0,5] \backslash\{1,2,3,4\} \\
& \triangle x\left(t_{k}\right)=\frac{1}{2} x\left(t_{k}^{-}\right), \quad t_{k}=k, \quad k=1,2,3,4 \\
& x(t)=e^{t+1}, \quad t \in\left[-\frac{1}{4}, 0\right] .
\end{aligned}
$$

Now by applying Theorem 3.2 we can prove that this system is controllable on [0,5]. Let us take

$$
\gamma=3 / 2, \quad \delta=1 / 2, \quad A=\left(\begin{array}{ll}
0 & 1 \\
0 & 0
\end{array}\right), \quad B=\left(\begin{array}{cc}
-1 & 2 \\
1 & 3
\end{array}\right), \quad C=\left(\begin{array}{l}
1 \\
2
\end{array}\right) .
$$

By computation we have

$$
\begin{aligned}
& c c^{*}=\left(\begin{array}{ll}
1 & 2 \\
2 & 4
\end{array}\right), \\
& E_{1,3 / 2} A(5-s)^{3 / 2}=\sum_{k=0}^{1} \frac{A^{k}(5-s)^{K 3 / 2}}{\Gamma(k+3 / 2)} \\
& =\frac{1}{\Gamma(3 / 2)}(I)+\frac{1}{\Gamma(5 / 2)}\left(\begin{array}{ll}
0 & 1 \\
0 & 0
\end{array}\right)(5-s)^{3 / 2}, \\
& E_{1,3 / 2} A^{*}(5-s)^{3 / 2}=\frac{1}{\Gamma(3 / 2)}(I)+\frac{1}{\Gamma(5 / 2)}\left(\begin{array}{ll}
0 & 0 \\
1 & 0
\end{array}\right)(5-s)^{3 / 2} .
\end{aligned}
$$


By simple calculation we can see that controllability matrix

$$
\begin{aligned}
W[0,5] & =\int_{0}^{5}(5-s)^{\frac{1}{2}}\left[E_{1,3 / 2} A(5-s)^{3 / 2}\right] c c^{*}\left[E_{1,3 / 2} A^{*}(5-s)^{3 / 2}\right] d s \\
& \approx(5)^{3}\left(\begin{array}{cc}
\frac{-3 \sqrt{5}-32}{\pi}-\frac{128(\sqrt[3]{5})}{81 \pi} & \frac{-16 \sqrt{5}-96}{3 \pi} \\
\frac{-16 \sqrt{5}-96}{3 \pi} & -\frac{32 \sqrt{5}}{3 \pi}
\end{array}\right) .
\end{aligned}
$$

As $w[0,5]$ is nonsingular, the conditions stated in Theorem 3.2 are satisfied. Hence the fractional system on $[0,5]$ is controllable.

Example 2 Consider the controllability of the following damped fractional differential system with state delay and impulses:

$$
\begin{aligned}
& { }^{c} D^{3 / 2} x(t)-\left(\begin{array}{ccc}
0 & 0 & 1 \\
0 & 1 & 0 \\
2 & 1 & -3
\end{array}\right){ }^{c} D^{1 / 2} x(t)=\left(\begin{array}{ccc}
1 & 1 & 1 \\
2 & 0 & 1 \\
2 & -1 & 2
\end{array}\right) x\left(t-\frac{\pi}{3}\right)+\left(\begin{array}{cc}
1 & 0 \\
1 & 1 \\
-1 & 2
\end{array}\right) u(t), \\
& t \in[0,3 \pi] \backslash\left\{\frac{\pi}{2}, \pi, \frac{3 \pi}{2}, 2 \pi, \frac{5 \pi}{2}\right\}, \\
& \triangle x\left(t_{k}\right)=\frac{1}{3} x\left(t_{k}^{-}\right), \quad t_{k}=k \frac{\pi}{2}, \quad k=1,2,3,4,5, \\
& x(t)=\tan t, \quad t \in\left[-\frac{\pi}{3}, 0\right] .
\end{aligned}
$$

Now by applying Theorem 3.3 we can prove that this system is controllable on $[0,3 \pi]$. Let us take

$$
\begin{aligned}
& \gamma=3 / 2, \quad \delta=1 / 2, \quad A=\left(\begin{array}{ccc}
0 & 0 & 1 \\
0 & 1 & 0 \\
2 & 1 & -3
\end{array}\right), \quad B=\left(\begin{array}{ccc}
1 & 1 & 1 \\
2 & 0 & 1 \\
2 & -1 & 2
\end{array}\right), \\
& C=\left(\begin{array}{cc}
1 & 0 \\
1 & 1 \\
-1 & 2
\end{array}\right) .
\end{aligned}
$$

Then we can obtain

$$
\begin{aligned}
& \operatorname{rank}\left[C|A C| \cdots \mid A^{n-1} C\right] \\
& \quad=\operatorname{rank}\left(\begin{array}{cccccc}
1 & 0 & 1 & 2 & \star & \star \\
1 & 1 & 1 & 1 & \star & \star \\
-1 & 2 & 6 & -5 & \star & \star
\end{array}\right)=3 .
\end{aligned}
$$

Thus by Theorem 3.3 this system is controllable on $[0,3 \pi]$.

\section{Conclusion}

We have evaluated controllability criteria for a damped fractional differential system with impulses and state delay. By using the step-by-step technique we constructed sufficient and necessary conditions for the controllability of such a system, which demonstrates that 
the controllability property is not dependent on impulses, delay, or fractional derivative order.

\author{
Acknowledgements \\ The authors would like to thank the editor and anonymous reviewers for their valuable comments and constructive \\ suggestions.
}

\title{
Funding
}

This work was supported by National Natural Science Foundation of China (110131013, 11471015).

\section{Availability of data and materials}

Not applicable.

Competing interests

The authors declare that they have no competing interests.

\section{Authors' contributions}

The authors contributed equally to the manuscript. All authors read and approved the final manuscript.

\section{Publisher's Note}

Springer Nature remains neutral with regard to jurisdictional claims in published maps and institutional affiliations.

Received: 26 March 2020 Accepted: 28 May 2020 Published online: 08 July 2020

\section{References}

1. Diethelm, K.: The Analysis of Fractional Differential Equations: An Application-Oriented Exposition Using Differential Operators of Caputo Type. Springer, Berlin (2010)

2. Hale, J.K., Lunel, S.M.V.: Introduction to Functional Differential Equations. Applied Mathematical Sciences. Springer, New York (1993)

3. Kilbas, A.A.A., Srivastava, H.M., Trujillo, J.J.: Theory and Applications of Fractional Differential Equations. Elsevier, Amsterdam (2006)

4. Miller, K.S., Ross, B.: An Introduction to the Fractional Calculus and Fractional Differential Equations. Wiley, New York (1993)

5. Podlubny, I.: Fractional Differential Equations: An Introduction to Fractional Derivatives, Fractional Differential Equations, to Methods of Their Solution and Some of Their Applications. Academic press, San Diego (1998)

6. Zheng, Z: Theory of Functional Differential Equations. Anhui Education Press, Hefei (1994)

7. Dauer, J.P., Gahl, R.D.: Controllability of nonlinear delay systems. J. Optim. Theory Appl. 21, 59-70 (1977)

8. Wei, J., Zuxiu, Z.: The general solution for the degenerate difference system with delay. Acta Math. Sinica (Chin. Ser.) 42, 769-780 (1999)

9. Jiang, W., Song, W.: Controllability of singular systems with control delay. Automatica 37, 1873-1877 (2001)

10. Wei, J.: Eigenvalue and stability of singular differential delay systems. J. Math. Anal. Appl. 297, 305-316 (2004)

11. Jiang, W.: Function-controllability of nonlinear singular delay differential control systems. Acta Math. Sinica (Chin. Ser.) 49, 1153-1162 (2006)

12. Jiang, W.: On the solvability of singular differential delay systems with variable coefficients. Int. J. Dyn. Syst. Differ. Equ. 4, 245-249 (2008)

13. Jiang, W.: The constant variation formulae for singular fractional differential systems with delay. Comput. Math. Appl. 59, 1184-1190 (2010)

14. Jiang, W.: The controllability of fractional control systems with control delay. Comput. Math. Appl. 64, 3153-3159 (2012)

15. Liu, L., Perc, M., Cao, J.: Aperiodically intermittent stochastic stabilization via discrete time or delay feedback control. Sci. China Inf. Sci. 62, 72201 (2019)

16. Balachandran, K., Dauer, J.P.: Controllability of nonlinear systems via fixed-point theorems. J. Optim. Theory Appl. 53 345-352 (1987)

17. Balachandran, K., Park, J.Y., Trujillo, J.J.: Controllability of nonlinear fractional dynamical systems. Nonlinear Anal. 75 1919-1926 (2012)

18. Balachandran, K., Govindaraj, V.L., Rodriguez-Germa, L., Trujillo, J.J.: Controllability of nonlinear higher order fractional dynamical systems. Nonlinear Dyn. 156, 33-44 (2013)

19. Dauer, J.P.: Nonlinear perturbations of quasi-linear control systems. J. Math. Anal. Appl. 54, 717-725 (1976)

20. Dai, L.: Singular Control Systems. Springer, Berlin (1989)

21. Duque, C., Uzcátegui, J.C., Leiva, H., Camacho, O.: Approximate controllability of semilinear strongly damped wave equation with impulses, delays, and nonlocal conditions. J. Math. Comput. Sci. 20, 108-121 (2019)

22. Jiang, W.: On the interval controllability of fractional systems with control delay. J. Math. Res. 9, 87 (2017)

23. Nirmala, R.J., Balachandran, K., Germa, L.R., Trujillo, J.J.: Controllability of nonlinear fractional delay dynamical systems. Rep. Math. Phys. 77, 87-104 (2016)

24. Zhou, X.F., Jiang, W., Hu, L.G.: Controllability of a fractional linear time-invariant neutral dynamical system. Appl. Math. Lett. 26, 418-424 (2013)

25. Rajagopal, J.N.: Relative controllability of nonlinear fractional delay dynamical systems with time varying delay in control. In: Theory and Applications of Non-integer Order Systems. Lect. Notes Electr. Eng., vol. 407, pp. 369-379. Springer, Cham (2017) 
26. Li, X., Liu, Z., Tisdell, C.C.: Approximate controllability of fractional control systems with time delay using the sequence method. Electron. J. Differ. Equ. 2017, 272 (2017)

27. Karthikeyan, S., Balachandran, K., Sathya, M.: Controllability of nonlinear stochastic systems with multiple time-varying delays in control. Int. J. Appl. Math. Comput. Sci. 25, 207-215 (2015)

28. Klamka, J.: Controllability of linear systems with delays in state and control. In: Controllability and Minimum Energy Control. Stud. Syst. Decis. Control., vol. 162, pp. 27-35. Springer, Cham (2019)

29. Bashirov, A.E., Kerimov, K.R.: On controllability conception for stochastic systems. SIAM J. Control Optim. 35, 384-398 (1997)

30. Bashirov, A.E., Mahmudov, N.I: On concepts of controllability for deterministic and stochastic systems. SIAM J. Control Optim. 37, 1808-1821 (1999)

31. Leiva, H., Merentes, N., Sanchez, J.: Interior controllability of the Benjamin-Bona-Mahony equation. J. Math. Appl. 33, 51-59(2010)

32. Matar, M.M.: On controllability of linear and nonlinear fractional integrodifferential systems. Fract. Differ. Calc. 9, 19-32 (2019)

33. Nawaz, M., Wei, J., Sheng, J., Niazi, A.U.K., Yang, L.: On the controllability of nonlinear fractional system with control delay. Hacet. J. Math. Stat. 49, 294-302 (2020)

34. Nawaz, M., Jiang, W., Sheng, J.: The controllability of nonlinear fractional differential system with pure delay. Adv. Differ. Equ. 2020, 183 (2020)

35. Nawaz, M., Wei, J., Jiale, S.: The controllability of fractional differential system with state and control delay. Adv. Differ. Equ. 2020, 30 (2020)

36. Bashirov, A.E., Ghahramanlou, N.: On partial approximate controllability of semilinear systems. Cogent Eng. 1, 965947 (2014)

37. Bashirov, A.E., Ghahramanlou, N.: On partial S-controllability of semilinear partially observable systems. Int. J. Control 88, 969-982 (2015)

38. Bashirov, A.E., Jneid, M.: On partial complete controllability of semilinear systems. Abstr. Appl. Anal. 2013, Article ID $521052(2013)$

39. Bashirov, A.E., Mahmudov, N., Semi, N., Etikan, H.: Partial controllability concepts. Int. J. Control 80, 1-7 (2007)

40. Leiva, H., Merentes, N., Sanchez, J.L.: Approximate controllability of semilinear reaction diffusion equations. Math. Control Relat. Fields 2, 171-182 (2012)

41. Leiva, H., Merentes, N., Sanchez, J.L.: A characterization of semilinear dense range operators and applications. Abstr. Appl. Anal. 2013, Article ID 729093 (2013)

42. Leiva, H., Merentes, N., Sanchez, J.L.: Controllability of the semilinear Benjamin-Bona-Mahony equation. J. Math. Appl. 35, 39-51 (2012)

43. Leiva, H., Merentes, N., Sanchez, J: Approximate controllability of a semilinear heat equation. Int. J. Partial Differ. Equ. 2013, Article ID 424309 (2013)

44. Leiva, H.: Rothés fixed point theorem and controllability of semilinear nonautonomous systems. Syst. Control Lett. 67 , 14-18 (2014)

45. Duque, C., Uzcátegui, J., Leiva, H.: Approximate controllability of semilinear dynamic equations on time scale. Asian J. Control 21, 2301-2307 (2019)

46. Leiva, H.: Robustness of the controllability for the strongly damped wave equation under the influence of impulses, delays and nonlocal conditions. Rev. Politec. 44, 33-40 (2019)

47. Leiva, H., Uzcategui, J:: Approximate controllability of discrete semilinear systems and applications. Discrete Contin. Dyn. Syst. 21, 1803-1812 (2016)

48. Leiva, H.: Controllability of semilinear impulsive nonautonomous systems. Int. J. Control 88, 585-592 (2015)

49. Leiva, H., Merentes, N.: Approximate controllability of the impulsive semilinear heat equation. J. Math. Appl. 38, 85-104 (2015)

50. Leiva, H.: Approximate controllability of semilinear impulsive evolution equations. Abstr. Appl. Anal. 2015, Article ID 797439 (2015)

51. Leiva, H., Sanchez, J.L.: Rothe's fixed point theorem and the controllability of the Benjamin-Bona-Mahony equation with impulses and delay. Appl. Math. 7, 1748 (2016)

52. Leiva, H.: Controllability of the impulsive functional BBM equation with nonlinear term involving spatial derivative. Syst. Control Lett. 109, 12-16 (2017)

53. Guevara, C., Leiva, H.: Approximated controllability of the strongly damped impulsive semilinear wave equation with memory and delay. IFAC J. Syst. Control 4, 1-6 (2018)

54. Carrasco, A., Leiva, H., Sanchez, J.L., Tineo, M.A.: Approximate controllability of the semilinear impulsive beam equation with impulses. Trans. IOT Cloud Comput. 2, 70-88 (2014)

55. Camacho, O., Leiva, H.: Impulsive semilinear heat equation with delay in control and in state. Asian J. Control 22 1075-1089 (2020)

56. Guevara, C., Leiva, H.: Approximated controllability of the strongly damped impulsive semilinear wave equation with memory and delay. IFAC J. Syst. Control 4, 1-6 (2018)

57. Selvi, S., Arjunan, M.M.: Controllability results for impulsive differential systems with finite delay. J. Nonlinear Sci. Appl. 5, 206-219 (2012)

58. Benchohra, M., Henderson, J., Ntouyas, S.: Impulsive Differential Equations and Inclusions. Hindawi Publishing Corporation, New York (2006)

59. Simeonov, P.: Impulsive Differential Equations: Periodic Solutions and Applications. Routledge, London (2017)

60. Fec, M., Zhou, Y., Wang, J.: On the concept and existence of solution for impulsive fractional differential equations. Commun. Nonlinear Sci. Numer. Simul. 17, 3050-3060 (2012)

61. Guo, T.L:: Controllability and observability of impulsive fractional linear time-invariant system. Comput. Math. Appl. 64, 3171-3182 (2012)

62. Vadivoo, B.S., Ramachandran, R., Cao, J., Zhang, H., Li, X.: Controllability analysis of nonlinear neutral-type fractional-order differential systems with state delay and impulsive effects. Int. J. Control. Autom. Syst. 16, 659-669 (2018) 
63. Zhang, H., Cao, J., Jiang, W.: Controllability criteria for linear fractional differential systems with state delay and impulses. J. Appl. Math. 2013, Article ID 146010 (2013)

64. Zhang, L., Ding, Y., Wang, T., Hu, L., Hao, K.: Controllability of second-order semilinear impulsive stochastic neutral functional evolution equations. Math. Probl. Eng. 2012, Article ID 748091 (2012)

65. Wan, X.J., Zhang, Y.P., Sun, J.T.: Controllability of impulsive neutral functional differential inclusions in Banach spaces. Abstr. Appl. Anal. 2013, Article ID 861568 (2013)

66. LV, X., Li, X., Cao, J., Perc, M.: Dynamical and static multisynchronization of coupled multistable neural networks via impulsive control. IEEE Trans. Neural Netw. Learn. Syst. 29, 6062-6072 (2018)

67. Garrappa, R., Popolizio, M.: Computing the matrix Mittag-Leffler function with applications to fractional calculus. J. Sci. Comput. 77, 129-153 (2018)

68. Balachandran, K., Govindaraj, V., Rivero, M., Trujillo, J.J.: Controllability of fractional damped dynamical systems. Appl. Math. Comput. 257, 66-73 (2015)

69. Cai, R., Zhou, H.-C., Kou, C.: The Kalman rank criterion for the controllability of fractional impulse controlled systems. IET Control Theory Appl. 14, 1358-1364 (2020)

70. He, B.-B., Zhou, H.-C., Kou, C.-H.: The controllability of fractional damped dynamical systems with control delay. Commun. Nonlinear Sci. Numer. Simul. 32, 190-198 (2016)

71. Zhang, X.: Some results of linear fractional order time-delay system. Appl. Math. Comput. 197, 407-411 (2008)

\section{Submit your manuscript to a SpringerOpen ${ }^{\circ}$ journal and benefit from:}

- Convenient online submission

Rigorous peer review

Open access: articles freely available online

High visibility within the field

- Retaining the copyright to your article

Submit your next manuscript at $\boldsymbol{~ s p r i n g e r o p e n . c o m ~}$ 